\title{
EL VILLANCET I EL TONO \\ DEL BARROC MUSICAL TARDIU EN FRANCESC VALLS. APORTACIÓ A L'ESTUDI D’AQUESTS GÈNERES MUSICALS
}

Josep PAVIA I Simó

\section{Resum}

El fet de conservar-se tants de Villancets i tants de tonos del mestre i compositor Francesc Valls, em va dur a contrastar els uns amb els altres, per tal d'esbrinar quines eren les coincidències i diferències d'aquests dos estils musicals, tan semblants entre si, pel que fa a la seva estructura, en els seus inicis. Tot seguit ofereixo el procès i el resultat del meu estudi, com a homenatge pòstum al meu mestre Miquel Querol i com una aportació més a l'estudi d'aquest tema.

\begin{abstract}
The fact that so many Villancets and Tones by the composer Francesc Valls $(* 1671 \mathrm{c} ; \dagger 1747)$ are preserved, lead me to check and contrast the formers with the latters, in order to guess which could be possible coincidences and differences between these two musical "styles", so similar in structure, at least in their beginning. Following, I will offer the process and results of my study, as a posthumous homage to my teacher, Dr. Miquel Querol, and also as a further contribution to the study of this subject.
\end{abstract}

No es tracta de fer un estudi del Barroc musical, ni del Villancet, ni del Tono, en un treball tan curt, com és aquesta petita col-laboració meva, precisament en l'homenatge al gran estudiós d'aquests temes i mestre meu, molt cordialment recordat, Miquel Querol.

Tan sols recordaré la definició que dóna Eugeni d'Ors, del Barroc en general ("Barroco es el elemento caótico del cosmos, el grito de la naturaleza desordenada, el movimiento de las pasiones. El espíritu barroco no sabe lo que quiere... El clasicismo es el mundo de las formas que pesan, el barroco, el de las formas que vuelan" (Citat per M. Querol). La qual definició, el propi Prof. Querol conduirà cap al Barroc musical i dirà que és una "Visión dinámica del mundo" que empeny a una renovació total. I considera la "'Dynamis" como "movimiento" y... como "fuerza". Esa "fuerza vital"... se traduce en arte por una exuberancia de formas nuevas, por una avidez de experiencias y de renovación total”. “... la manifestación más auténtica del barroco la constituye la música, arte por excelencia del movimiento y de las formas que vuelan".

D'altra banda, remarcar també, des d'un principi, la importància que té la prolífica producció del nostre gran mestre Francesc Valls en aquests temes. Serà bo recordar que, a la Biblioteca de 
Catalunya, principalment al Fons Musical de la Catedral de Barcelona, es conserven uns cent vint-i-set Villancets d'aquest compositor amb música i, a la mateixa Biblioteca, també es conserven una cinquantena de $\operatorname{Tonos}^{1}$, material suficient per a bastir el meu propòsit. A l'inventari de donació que fa Valls de les seves composicions a la Catedral de Barcelona, hom llegeix, grosso modo, la següent relació de paquets de Villancets i de Tonos:

- "29 Villancicos ab Viols., a 8, 9 y 10, de N. a S. a "21 Villancicos ab Viols., a 8, 9 y 10, del SSm. Sagt."; "20 Villancicos, a 10 y a 12, al SSm. Sagt."; "13 Villancicos, a 10 y a 12, a N. " S. a, "12 Villancicos, a dos cors, al SSm. Sagt."; "4 Villancicos a dos cors, a N. a S."; "4 Villancicos per qualsevol Sant, ab Viols."; "40 Villancicos de S. Thomás ab minist., altres ab Viols.”; "Villancicos al Sgt. y a N. S., ab minist. y altres ab Viols".

- "54 tonos, ab Viols. Al Sagt. y a N. ${ }^{a}$ S. a, a duo y solo"; "16 tonos, a 4 y a 3, al SSm Sagt. y a N. ${ }^{\text {a }}$ S. a"; "29 tonos a solo y a duo, al SSm. y a N. ${ }^{\text {a }}$ S. a; i "27 tonos ab Viols., al SSm. Sagt. y a N. ${ }^{a}$ S. ${ }^{a}$, a $4 "$ ".

- A part d'això, cal afegir un llistat d'uns seixanta Villancets, el text dels quals - no la música- es conserva als Fons Bonsoms i Fons Aguiló de la mateixa Biblioteca de Catalunya i jo mateix n'he publicat les referències ${ }^{2}$. El mèrit d'aquesta seixantena de Villancets rau en el fet que la major part d'ells no estan a la llista d'aquells, dels quals es conserva la música i en conseqüència, n'augmenta notòriament el nombre.

Pel que fa al present treball, confesso que, a més de l'homenatge al Mestre, respon a un desig d'aclariment i, també, d'aprofundiment en el tema que l'encapçala.

Fa uns pocs mesos, en una entrevista radiada que se'm féu a Catalunya Música, per un dels fantàstics entrevistadors i conductors dels programes musicals d'aquesta emisora, en Raimon Colomer, al programa "Vistes al mar", dedicat en aquella ocasió al mestre Francesc Valls, el susdit Colomer, em preguntà si podia diferenciar entre Villancico i Tono i, en una resposta, pot ser massa agosarada i optimista, li vaig respondre afirmativament, pensant matisar-ho amb l'explicació subsegüent. Però el discurs, de vegades, fa que t'allunyis d'allò que volies dir i ja no hi tornes. Tanmateix, per les dades que vaig donar, es podia deduir, que no estava tan clar. Per això, ara, aprofito aquesta oportunitat per matisar aquella afirmació. A l'escrit, es dominen millor tots els caps. A més, també confesso que el tema em seduí de debò. En el resultat, doncs, de les meves anàlisis, hom hi trobarà uns detalls, en els quals coincideixen ambdós gèneres, d'altres que els fan diferents, i d'altres que plantegen més interrogants. Per tant, crec que encara cal esperar una resposta definitiva.

Amb la susdita entrevista, es pretenia donar a conéixer al públic en general la figura del nostre gran compositor i teòric, conegut principalment pel món especialitzat de la musicologia, però no tant per la resta de l'afició musical del nostre poble, ben cert perquè la seva obra no els ha arribat, a travès de con-

1. Pavia I Simó, Josep: Tonos de Francesc Valls (1671c-1747). Vol. I. Barcelona, Consejo Superior de Investigaciones Científicas, "Monumentos de la Música Española, vol. 58", 1999. 303 pp. ISBN: 84-00-07847-0; ID, Vol. II. Barcelona, Consejo Superior de Investigaciones Científicas, "Monumentos de la Música Española, vol. 64”, 2001. 288 pp. ISBN: 84-00-07954-0.

2. Pavia i Simó, Josep: La Música en Cataluña en el siglo XVIII. Francesc Valls (1671c-1747). Barcelona, Consejo Superior de Investigaciones Científicas, “Monumentos de la Música Española, vol. 53”, 1997. 501 pp. ISBN: 84-00-07700-8. 
certs i publicacions, degut al fet que la música de Valls no ha estat tan considerada com la música d'altres contemporanis seus del món i no s'ha recuperat i divulgat suficientment. El nostre mestre Francesc Valls, però, no té perquè avergonyir-se, si el posen al costat d'altres compositors de primera fila.

Tanmateix, ara, no em proposo repetir el que d'altres estudiosos i jo mateix hem publicat sobre Valls, sinó analitzar i comparar els gèneresVillancico i Tono en l'obra d'aquest compositor, ja que, pel que consta, en fou un autor fecund, per tal que el resultat d'aquesta comparació, afegit a d'altres estudis semblants que s'han fet i s'estan fent, ajudi a entendre millor aquests dos gèneres que, en la seva arrel estructural, no es diferencien tan profundament entre ells. En realitat, ambdós gèneres constaven d'Estribillo i Coplas, a l'inici. I els afegitons d'altres apartats, també afecten, més o menys, tots dos, com s'anirà comprobant. Analitzaré, doncs, les convergències i les diferències.

\section{El Villancet i el Tono del Barroc musical tardiu en Francesc Valls}

Per tal de dur a terme aquest estudi, m'he proposat, en aquesta ocasió, fer la comparació entre 39 Villancicos i 39 Tonos, per servar una proporció. En una altra oportunitat, intentaré fer l'estudi de la resta de Villancicos i Tonos d'aquest autor.

Valls va compondre, quasi bé en la seva totalitat, música per al culte de la Catedral de Barcelona, de la qual n'era el Mestre de Capella. Una gran part era en llatí, per als textos litúrgics de la Missa i del Breviari, dels quals, uns són invariables o de l'ordinari (Pater noster, Kyries, Gloria, Credo, Sanctus i Agnus) i d'altres, variables (Introitus, Offertorium, Comunio, Psalmi, Responsoria, etc. i són diferents cada dia), els quals encara poden ser, o del propi, per a les festivitats que en tenen de particular i del comú, per a festivitats que no en tenen.

Valls també va compondre altra música no litúrgica, religiosa i profana. I aquí trobem els Villancets i els Tonos.

Seguint, doncs, la línia dels estudis que s'han fet fins ara, sobre els gèneres Villancet i Tono, he analitzat l'obra de Valls i hi trobo el següent:

Metodologia. Després d'uns aclariments previs referents als conceptes, donaré en forma d'esquemes els diferents aspectes del treball, tot remetent a ells, des dels apartats precedents, de manera que es pugui comparar fàcilment allò que s'ha anat dient del Villancet i del Tono. Les notes al peu de pàgina explicaran els detalls que calgui posar de relleu, en cada moment.

El nom. A l'hora de cercar les convergències i/o divergències entre els dos gèneres musicals que m'ocupen, trobo una primera diferència irrebatible que s'observa en el nom. Tots els compositors han establert sempre d'una manera ben clara, quan volien compondre un "Villancico" i quan un "Tono". Només he trobat una composició de Valls que, al Fons Bonsoms (abreujat: FB) s'anomena "Villancico" i a la coberta de la pròpia composició, "Tono"3. Sense tenir en compte aquesta excepció, i pensant que confirma la regla, caldrà concloure, que el nom és clarament diferenciador.

3. M 1611/III; FB 1186/III i 3283/III. El text és el mateix a tots tres. 
Signatura, Títol, Nombre de veus i Destinació. Donaré la referència del Catàleg del RISM per als Villancets i Tonos que hi estan catalogats i la signatura corresponent de la Biblioteca on es troben, precedits d'un número, ordinació artificial per a aquest treball, perquè resulti més còmoda i fàcil la referència.

El títol, el nombre de veus i la destinació són els que consten a la portada de cada manuscrit. Vid. Esquema A) i Esquema B).

Pel que fa a la destinació, els musicòlegs han deduit, dels seus estudis, que, la diferència de nom és en vistes a la destinació, això és, els Villancets són de caràcter religiós i sembla que no s'hi ha trobat cap excepció. Els Tonos, poden ser de caràcter religiós (o "a lo divino") i/o profà ("a lo humano"), essent aquests darrers altament majoritaris i quasi bé en exclusiva, durant alguns segles. Però, en l'obra analitzada de Valls, corresponent al Barroc tardiu, la proporció és inversa, ja que de 39 Tonos estudiats, només 7 són "a lo humano", com es comproba a l'esquema B). El còmput dels Tonos de Valls, que es conserven a la Biblioteca de Catalunya, i que jo mateix he publicat (loc. cit. més amunt), arriba a una cinquantena i són la resta dels 126 que el propi autor havia donat a la Catedral, quan es va jubilar.

Altre detall curiós que he observat és que, en tota la relació de Follets dels Fons Bonsoms (223 exemplars, algun repetit) i Fons Aguiló (191, en tres volums, encara que 32 del volum tercer no són musicals), conservats a la Biblioteca de Catalunya i que també he publicat, no apareix cap Tono, com si els mateixos "Tonos a lo Divino" estiguessin exclosos de l'ús religiós. Aquests Follets recullen les notícies sobre la celebració de les festes a Catalunya i els textos literaris de les composicions musicals: Villancets, Oratoris, Drames, etc., que s'estrenaven en cada festa, entre els quals, com acabo de dir, no s'hi troba cap Tono de Valls.

L'estructura formal. L'estructura formal del Villancet i del Tono, inicialment i bàsica, és la mateixa, car ambdós gèneres estan formats per l' Estribillo i les Coplas. De tal manera que, dels 39 Villancets i Tonos que he analitzat de Valls, dels existents a la Biblioteca de Catalunya, hom troba 22 Villancets i 26 Tonos, amb l'estructura E-C. Després, s'hi aniran introduint, segons els gustos, pot ser per influència italiana, altres apartats, com és la "Introduccion”, "el Recitativo", l"'Ària", la "Responsion", etc. a tots dos gèneres, amb diferents combinacions d'aquests elements. Així, doncs, de vegades, eliminaven l'Estribillo i/o les Coplas; d'altres, repetien, alternativament, l'Estribillo, la Copla, l'Aria i el Recitativo, fins i tot a cinc vegades, aproximant-se, conscientment o inconscient, a la Cantata i d'altres moviments contemporanis. La diferència, doncs, no sembla tan evident, tampoc, a partir de l'estructura formal. Vid. Esquema C) i Esquema D)

Instruments, Distribució de Veus i Acompanyaments. També és sorprenent, la diferència que fa Valls, en l'ús d'aquests tres apartats. Pel que fa als instruments i als acompanyaments, la diferència és remarcable, perquè, mentre als Tonos hi surten exclusivament violins, als Villancets hom hi troba violins, xeremies i clarins. Això, parlant d'instruments solistes, perquè en els d'acompanyament, també hi ha una diferència notòria. Així, doncs, mentre Tonos i Villancets empren el violó / acompanyament als violins i l'arpa (aquesta, surt a un Tono i a un Villancet), el "sacapuche”, no surt a cap Tono i sí als 
Villancets. L'orgue tampoc no surt a cap Tono, però sí a 30 Villancets. També és comú l'acompanyament continu, però no a totes les peces. I quant a les veus, els Tonos van d'una a set veus, sense que n'hi hagi cap de sis, a la Biblioteca de Catalunya, però sí que n'hi ha un al manuscrit de Cracòvia ("Tono a 6 con Violines al Nac. ${ }^{\text {to }}$ de la Virgen, Ha de la coriente", fol. 63) 4 i els Villancets, de sis a divuit veus. No baixa de sis. Vid. Esquema E).

Nombre de Veus. Distribució de les veus en Cors i dels Instruments El primer Cor sol rebre un tractament especial, quant a la distribució de les veus, ja que, quan n'hi ha més, acostuma duplicar alguna de les veus i, aleshores, desapareix el Baix. Cal remarcar que sempre ocorre en el primer coro, als Villancets. Potser la desaparició del Baix era deguda al fet que aquesta part la solia fer un instrument i el compositor volia que, al menys, al primer Cor, fossin tot veus humanes. De vegades, només li deixen dues veus, a gust del compositor. El 4. ${ }^{\circ}$ Coro també solia ser instrumental, o de "ministriles".

Podria influir la tonalitat? Crec que tampoc massa, perquè, en general, estan representades totes les tonalitats, tant als Villancets, com als Tonos, com es comprobarà més endavant. Vid. Esquema F) i Esquema L)

Podria influir la mètrica literària en la diferenciació del Villancet i del Tono? No sabria dir fins a quin punt. Perquè cada part (Estribillo, Introducción, Coplas, Aria, etc.) sol tenir el mateix tractament mètric, en els Villancets i en els Tonos. Només cal comparar els textos, per veure quines parts tenen tractament estròfic, o de romanç5.

\section{Esquema A) Villancets}

\begin{tabular}{ccllll} 
N. $^{\circ}$ & N. $^{\mathbf{R}}$ RSM & Sign. $\boldsymbol{E}: \boldsymbol{B} \boldsymbol{b} \boldsymbol{c}$ & Títol & Veus & Dedicatòria \\
\hline $1^{6}$ & 00072 & M1470/2: & Clarines de la fama. & a 16v. & Entrada de Rei \\
2 & 00073 & M1470/5: & Quien será dezid. & a 12v. & Immaculada \\
3 & 00313 & M1470/6: & Ya del Sol de Gracia. & a 14v. & Para un velo \\
4 & 00133 & M1470/7: & Hermosas Ninfas de Fetis. & a 10v. & Para un velo \\
5 & 00123 & M1470/8: & Esferas, regiones. & a 15v. & St. Ramon/St. T. Aquino \\
6 & 00282 & M1470/9: & Sagrado Padre. & a 7v. & Immaculada \\
7 & 00011 & M1470/12: & A ver la Competencia. & a 6v. & S.mo S.to. \\
8 & 00047 & M1471/1: & Aquella admirable zarza & a 12v. & S.mo S.to. \\
9 & 00075 & M1471/2 & Con nuevos aplausos & a 6v. & S.mo S.to.
\end{tabular}

4. Manuscrit de Cracòvia, PL: $K j$. Les composicions que analitzo d'aquest manuscrit, no hi són, al RISM. ("Répertoire International des Sources Musicals").

5. Es comprén, per l'espai disponible, que no puc incloure els textos literaris dels Villancets i dels Tonos, per poder-ne fer la comparació. L'anàlisi comparativa dels mateixos, la deixo per a una altra ocasió, per poder-ne donar una visió més ampla.

6. Afegeixo aquesta numeració ordinal, per facilitar les referències posteriors. Així, doncs, em referiré a un Villancet, escribint aquest número ordinal i el que li correspon, dins del paquet de la signatura corresponent, per ex.: V1 = 1470/2 ... I a un Tono, dient: $\mathrm{T} 1=1470 / 13$. 


\begin{tabular}{|c|c|c|c|c|c|}
\hline 10 & 00232 & M1471/3: & Oiga el que mató la muerte, & a $10 \mathrm{v}$. & S.mo S.to. \\
\hline 11 & 00045 & M1471/5: & Animada armonia, & a $9 v$ & A Nra. Sra. \\
\hline 12 & 00209 & M1471/8: & Misterio soberano, & a $12 \mathrm{v}$. & S.mo S.to. \\
\hline 13 & 00142 & M1471/11: & Huyendo el Profeta Elias, & a $12 \mathrm{v}$. & S.mo S.to. \\
\hline 14 & -7 & M1471/13: & Ha del centro infeliz de los pesares, & a $11 \mathrm{v}$. & Concepcion \\
\hline 15 & - & M1471/14: & Pues la razón no alcanza, & a $12 \mathrm{v}$. & S.mo S.to. \\
\hline 16 & - & M1471/15: & Enfermó Adán y sus hijos, & a $11 \mathrm{v}$. & S.mo S.to. \\
\hline 17 & - & M1471/16: & De un transparente cristal, & a $15 \mathrm{v}$. & S.mo S.to \\
\hline 18 & 00037 & M1471/17: & Alados Serafines, & a $14 \mathrm{v}$. & Sto. Tomas Aquino \\
\hline 19 & - & M1471/18: & Ola hau Pastores, & a $12 \mathrm{v}$. & SS. Reyes \\
\hline 20 & - & M1471/19: & Para lograr que la Gracia, & a $12 \mathrm{v}$. & S.mo S.to. \\
\hline 21 & 00256 & M1471/20: & Que Assombro! & a $12 \mathrm{v}$. & Immaculada \\
\hline 22 & - & M1471/21: & Navecilla que llevas, & a $10 v$. & S.mo S.to. \\
\hline 23 & 00019 & M1472 /7: & Ha de la lobrega estancia, & a $12 \mathrm{v}$. & S.mo S.to. \\
\hline 24 & 00231 & M1472 /8: & O misterioso cariño, & a $15 \mathrm{v}$. & S.mo S.to. \\
\hline 25 & 00254 & M1472 /9: & Pues que con quatro palabras, & a $16 v$. & S.mo S.to. \\
\hline 26 & 00040 & M1472 /10: & Amantes Venid, & a $16 \mathrm{v}$. & S.mo S.to. \\
\hline 27 & 00260 & M1472 /11: & Qué Metrico Acento, & a $16 \mathrm{v}$. & S.mo S.to. \\
\hline 28 & - & M1472 /12: & Que feliz accento, & a $12 \mathrm{v}$. & Dedic. S.mo S.to. \\
\hline 29 & 00036 & M1472 /13: & Al triumfo sagrado, & a $13 \mathrm{v}$. & S.mo S.to. \\
\hline 30 & 00246 & M1472 /14: & Potencias y sentidos, & a $13 \mathrm{v}$. & Corpus en... S. Juan \\
\hline 31 & 00281 & M1472 /15: & Rompan del Vientos las Vagas Espheras & , a $12 \mathrm{v}$. & S.mo S.to. \\
\hline 32 & - & M1472 /16: & Ha del Pastor, & a $6 v$ & Dedic. S.mo S.to. \\
\hline 33 & 00115 & M1472 /17: & En los diafanos espacios, & a $11 \mathrm{v}$. & S.mo S.to. \\
\hline 34 & 00317 & M1472 /18: & Ya se huyeron las sombras Antiguas, & a $12 \mathrm{v}$. & S.mo S.to. \\
\hline 35 & - & M1472 /19: & Ha de la ardiente hoguera, & a $9 v$ & Dedic./ S.mo S.to. \\
\hline 36 & 00303 & M1472 /20: & Trinad Avezillas, & a $8 \mathrm{v}$ & S.mo S.to.. \\
\hline 37 & - & M1473 /9: & Alarma. & a $8 \mathrm{v}$. & A S. Rafael \\
\hline 38 & 00067 & M1473 /10: & Cathalana agraciada. & a 6. & A S. Eulalia \\
\hline 39 & 00034 & M1474 /18: & $\mathrm{Al}$ son de los Cl.s y timb.s. & a $18 \mathrm{v}$. & SS Reyes S.mo S.to \\
\hline
\end{tabular}

Esquema B) Tonos.

\begin{tabular}{rrllll} 
N. $^{\circ}$ & N. $^{\circ}$ RISM & Sign. $\boldsymbol{E}: \boldsymbol{B b c}$ & Títol & Veus & Dedicatòria \\
\hline 1 & 00160 & M1470/13 & La tierra el viento. & a 4 & S. Fco. Javier \\
2 & 00128 & M1611/26 & Filomenas del Golfo. & a 5 & Santísimo Sacramento \\
3 & 00093 & M1611/27 & Del altar en las playas. & a solo & Santísimo Sacramento \\
4 & 00158 & M1611/28 & La perla preciosa. & a 4 & Immaculada \\
5 & 00057 & M1611/29 & Ay amor quan dulcemente. & a 4 & S.mo Sacramento \\
6 & 00001 & M1611/30 & A Dios amante suspiran. & a 4 & S.mo Sacramento, \\
7 & 00035 & M1611/31 & Al templo mas elevado. & a $~$ & Santísimo Sacramento \\
8 & 00050 & M1611/33 & Ausente de tus ojos divino echizo. & Al Humano \\
9 & 00032 & M1611/34 & Al Dios que amoroso. & a duo & S.mo Sacramento \\
10 & 00151 & M1611/36 & Jilguerillo que el aire. & a solo & Al Humano
\end{tabular}

7. Els Villancets i Tonos sense numeració del RISM, vol dir que no es troben en aqueixa catalogació. 


\begin{tabular}{|c|c|c|c|c|c|}
\hline 11 & 00213 & M1611/37 & Ninfas que en bosques sagrados. & a 4 & Al Humano \\
\hline 12 & 00079 & M1611/38 & Quando Antonio glorioso. & a 4 & San Antonio \\
\hline 13 & 00046 & M1611/39 & Aquel Astro que luze. & a 4 & San Joseph \\
\hline 14 & 00299 & M1611/40 & Tienes de engolfarte en penas. & a 4 & Para un Velo \\
\hline 15 & 00009 & M1611/41 & A todo correr. San Antonio, & a 4. & S. Ant ${ }^{o}$. \\
\hline 16 & 00124 & M1611/42 & Espiritu ardiente en llamas. & a 3 & S.mo Sacramento \\
\hline 17 & 00283 & M1611/438 & Sagrado portento. & a 4 & S.mo Sacramento \\
\hline 18 & 00255 & M1637/VIII/8 & Pura brillante Aurora. & a solo & Immaculada \\
\hline 19 & 00253 & M1637/VIII/9 & Pues oy benignas las luzes. & a solo & Immaculada \\
\hline 20 & 00092 & M1686/26 & [Deidades del abismo] & a duo & Para un Velo \\
\hline 21 & 00300 & M1686/27 & Toda hermosa es la Madre del Sol. & a solo & Immaculada \\
\hline 22 & 00304 & M1686/28 & Triunfante de la muerte. & a 5 & Assumpció? de Maria \\
\hline 23 & 00239 & M1686/29 & Para q. no se malogre. & a 4 & San Antonio \\
\hline 24 & 00060 & M1686/30 & Ay que prodigio. & a 4 & San Joseph \\
\hline 25 & 00120 & M1686/31 & Entre rosicler la Aurora. & a solo & Al Humano \\
\hline 26 & 00095 & M1686/32 & Descansad, dormid. & a 4 & San Joseph \\
\hline 27 & 00270 & M1686/33 & Quien de Joseph. & a 4 & San Joseph \\
\hline 28 & 00159 & M1686/34 & La que en el jardin. & a 4 & San Antonio \\
\hline 29 & 00271 & M1686/35 & Quien de Maria en las glorias. & a duo & Immaculada \\
\hline 30 & 00110 & M1686/36 & En aplauso feliz. & a 7 & Immaculada \\
\hline 31 & 00105 & M1686/37 & Dulce apacible. & a 3 & Al Humano \\
\hline 32 & 00114 & M1686/38 & En la Eminente Cima. & a solo & Al Humano \\
\hline 33 & 00259 & M1686/39 & Que estruendo. & a 4 & San Antonio \\
\hline 34 & 00113 & M1686/40 & [En festivos aplausos]. & a 4 & San Antonio \\
\hline 35 & 00314 & M1686/41 & Ya el Cielo puede. & a 4 & Al Humano \\
\hline 36 & 00112 & M1686/43 & En el misterioso circo. & a 7 & Santísimo Sacramento \\
\hline 37 & 00240 & M1686/44 & Paranimfos celestiales. & a 7 & Santísimo Sacramento \\
\hline 38 & - & M1690/29 & Nuevo concurso de luces & a 3 con & s $A S . A n t^{o}$ \\
\hline 39 & - & PL: $K j$, f. 7 & Sacro atractivo del alma & a 4 & S.mo S.to. \\
\hline
\end{tabular}

\section{Esquema C) Estructura formal . I) dels Villancets}

\begin{tabular}{cll} 
N. $^{\mathbf{0}}$ RISM & Sign. $\boldsymbol{E}: \boldsymbol{B} \boldsymbol{b} \boldsymbol{c}$ & Estructura $^{10}$ \\
\hline- & M1473 /9: & ??? \\
00073 & M1470/5: & (E) - C \\
00313 & M1470/6: & (E) - C 11 \\
00133 & M1470/7: & (E) - C
\end{tabular}

8. Estructura més aviat semblant a la Cantata.

9. Aquesta Signatura es diu: "M 1690/2 Tonos" i conté diferents Tonos incomplets. Al Tono que m'ocupa, sembla que només li manca el Tiple.

10. Per a les diferents seccions o moviments dels Villancets i Tonos, empraré les llegendes següents: $\mathrm{A}=$ Ària; $\mathrm{C}=$ Copla/Coplas, en català: Cobla/Cobles); E = Estribillo; I = Introducción; R = Recitado, Responsión i/o Respuesta; T = Todos. Igualment, per a designar les veus, els instruments i les Biblioteques, ho faré amb les sigles del RISM. Així, doncs, les veus aniran amb les inicials en majúscula (advertint, però, que amb l' "S" em referiré al "Tiple", amb la "A" a l' "Alto", amb la "T" al "Tenor" i amb la "B" al "Bajo"), els instruments les portaran en minúscula i, quant a les Biblioteques: $E$ : $B b c=$ España:Barcelonabibliotecacentrral; $P L . K j=$ Polònia: CracòviaUniversitat. L'interrogant: ? = manuscrit incomplet o altres causes. 11. Apareixen els termes "Ayre", "aprisa", "despacio". 


\begin{tabular}{cll}
00123 & M1470/8: & E - C \\
00011 & M1470/12: & E - C \\
- & M1471/13: & E - C \\
- & M1471/14: & E - C \\
00037 & M1471/17: & E - C \\
- & M1471/18: & E - C \\
- & M1471/21: & E - C \\
00019 & M1472 /7: & E - C \\
00040 & M1472 /10: & E - C \\
00260 & M1472 /11: & E - C \\
- & M1472 /12: & E - C \\
00036 & M1472 /13: & E - C \\
00115 & M1472 /17: & E - C \\
00303 & M1472 /20: & E - C \\
00067 & M1473 /10: & E - C \\
00246 & M1472 /14: & E - C - R \\
- & M1472 /19: & E - R - C \\
00072 & M1470/2: & I - E - C 13 \\
00142 & M1471/11: & I - E - C \\
- & M1471/15: & I - E - C 14 \\
\hline- & M1471/16: & I - E - C \\
00254 & M1471/19: & I - E - C 15 \\
00282 & M1472 /9: & I - E - C \\
00231 & M1470/9: & I - E) - C \\
00075 & M1472 /8: & I - R - T \\
00045 & M1471/2: & A - R - C - T \\
00209 & M1471/5: & E - R - A - C \\
00047 & M1471/8: & E - R - A - C \\
00256 & M1471/1: & I - E - C - R \\
- & M1471/20: & I - E - R - A \\
00317 & M1472 /16: & E - C - T - R - A \\
00281 & M1472 /15: & E - R - A - R - T \\
00034 & M1474 /18: & E - R - C - R - A \\
00232 & M1471/3: - R - A \\
& & I - E - R - A - R - A \\
& & \\
\hline- & &
\end{tabular}

12. "a 16". També a d'altres.

13. En general, a tots els Villancets, les Cobles es reparteixen entre les diferents veus. En aquest, però, de la forma següent: Cobla 2..$^{\text {, }}$ S 1; Cobla 1. a i 4. a , S 2; Cobla 3. a , T. A diferents veus i instruments i en lloc de dir: "Estribillo", diu: "Despues de la Introduccion". Les Cobles tenen una "Respuesta" cantada per "Todos" i els "Bajos" que no tenen text, els poso en minúscula, entenent que devien estar interpretats per un instrument.

14. "eco", "espacio", "con ayre".

15. "a solo y a quatro", com en molts altres villancets. 


\section{Esquema D) Estructura formal. II) Dels Tonos}

\begin{tabular}{|c|c|c|}
\hline N. ${ }^{\circ}$ RISM & Sign. $E: B b c$ & Estructura \\
\hline 00092 & M1686/26 & $E-C^{16}$ \\
\hline 00300 & M1686/27 & $E-C$ \\
\hline 00304 & M1686/28 & $E-C^{17}$ \\
\hline 00158 & M1611/28 & $E-C^{18}$ \\
\hline 00046 & M1611/39 & $E-C^{19}$ \\
\hline 00060 & M1686/30 & $E-C^{20}$ \\
\hline 00270 & M1686/33 & $E-C^{21}$ \\
\hline 00255 & M1637/VIII/8 ? & $?-\mathrm{C}^{22}$ \\
\hline 00093 & M1611/27 & $E-C$ \\
\hline 00079 & M1611/38 & $E-C^{23}$ \\
\hline 00299 & M1611/40 & $?-\mathrm{C}^{24}$ \\
\hline 00057 & M1611/29 & $E-C^{25}$ \\
\hline 00001 & M1611/30 & $E-C$ \\
\hline 00032 & M1611/34 & $E-C$ \\
\hline 00124 & M1611/42 & $E-C^{26}$ \\
\hline 00253 & M1637/VIII/9 & $E-A-C$ \\
\hline 00120 & M1686/31 & E - C \\
\hline 00050 & M1611/33 & $E-C$ \\
\hline 00151 & M1611/36 & $E-C$ \\
\hline 00114 & M1686/38 & $E-C$ \\
\hline 00128 & M1611/26 & $E-C^{27}$ \\
\hline 00213 & M1611/37 & $E-C^{28}$ \\
\hline 00035 & M1611/31 & $E-C^{29}$ \\
\hline 00110 & M1686/36 & $E-C$ \\
\hline 00314 & M1686/41 & $E-C$ \\
\hline 00112 & M1686/43 & $E-C$ \\
\hline 00240 & M1686/44 & $\mathrm{E}-\mathrm{C}$ \\
\hline - & PL: $K j$, f. 7 & $E-C^{30}$ \\
\hline 00271 & M1686/35 & $\mathrm{E}-\mathrm{C}-2 .^{\circ} \mathrm{E}$ \\
\hline 00105 & M1686/37 & $E-C-R^{31}$ \\
\hline 00009 & M1611/41 & $E-C^{32}-R$ \\
\hline
\end{tabular}

16. Cobles $=\mathrm{S} 1,2$, acompanyament.

17. Cobles $=$ vl 1, 2, violón, S, T, acompanyament.

18. Entre el S 1 i el $S 2$.

19. Alternatim S 1 i T.

20. Cobles amb "Solos", per les diferents veus.

21. Estribillo i Cobles: Solos i polifonia.

22. Incomplet.

23. Cobles 1. ${ }^{\mathrm{a}}$ i $2 .^{\mathrm{a}}=\mathrm{S} 1+\mathrm{duo}$; "Respuesta a las Coplas" = S 1 i A; Cobles 2." i 4. ${ }^{\mathrm{a}}=\mathrm{T}$.

24. Incomplet.

25. Cobles així: S, S, A, A.

26. Cobles $=\mathrm{A}$

27. Cobles $1 .^{\mathrm{a}}, 3$. $^{\mathrm{a}}$ i $5 .^{\mathrm{a}}=\mathrm{S} 1$; C $2 .^{\mathrm{a}}$ i $4 .^{\mathrm{a}}=\mathrm{S} 2$.

28. Cobles = una cada veu, excepte l'A que no en fa cap.

29. Cobles $=$ una cada veu.

30. S 1 (1. ${ }^{\mathrm{a}}$ part $)+$ veus.

31. Cobles i Recitatiu $\left(1 .^{\mathrm{a}}\right.$ part $)=$ Cupido. 


$\begin{array}{lll}00113 & \mathrm{M} 1686 / 40 & \mathrm{E}-\mathrm{R}-\mathrm{C} \\ 00095 & \mathrm{M} 1686 / 32 & \mathrm{E}^{33}-\mathrm{R}^{34}-\mathrm{A}^{35}-\mathrm{C}^{36} \\ 00259 & \mathrm{M} 1686 / 39 & \mathrm{E}-\mathrm{R}^{37}-\mathrm{A}-\mathrm{R}^{38}-\mathrm{T} \\ 00239 & \mathrm{M} 1686 / 29 & \mathrm{E}-\mathrm{R}^{39}-\mathrm{C}^{40}-\mathrm{R}^{41}-\mathrm{A}^{42} \\ 00159 & \mathrm{M} 1686 / 34 & \mathrm{E}-\mathrm{C}^{43}-\mathrm{T}-\mathrm{R}^{44}-\mathrm{A}^{45} \\ 00160 & \mathrm{M} 1470 / 13 & \mathrm{I}-\mathrm{R}-\mathrm{A}-\mathrm{R}-\mathrm{A} \\ 00283 & \mathrm{M} 1611 / 43^{46} & \mathrm{E}-\mathrm{R}-\mathrm{postR}-\mathrm{A}-\mathrm{R}-\text { postR }-\mathrm{A} \\ - & \mathrm{M} 1690 / 2 & \mathrm{I}-\mathrm{E}-\mathrm{R}-\mathrm{A}-\mathrm{C}-\mathrm{R}-\mathrm{A}-\text { Todos }\end{array}$

\section{Esquema E) Instruments, Distribució de Veus i Acompanyaments}

\section{I) Villancets}

\begin{tabular}{|c|c|c|c|c|c|}
\hline N. ${ }^{\circ}$ RISM & Sign. $E: B b c$ & Instrum. $^{\mathrm{s}}$ & Veus & & \\
\hline 00072 & M1470/2: & vl 1,2 xeremia 1 & $\mathrm{~S} 1,2, \mathrm{~A}, \mathrm{~T}$ & $\mathrm{~S}, \mathrm{~A}, \mathrm{~T}, \mathrm{~b}^{47}$ & $\mathrm{~S}, \mathrm{~A}, \mathrm{~T}, \mathrm{~b}$ \\
\hline 00073 & M1470/5: & & $\mathrm{S} 1,2, \mathrm{~A}, \mathrm{~T}$ & $\mathrm{~S}, \mathrm{~A}, \mathrm{~T}, \mathrm{~b}$ & $\mathrm{~S}, \mathrm{~A}, \mathrm{~T}, \mathrm{~b}$ \\
\hline 00313 & M1470/6: & & $\mathrm{S} 1,2, \mathrm{~A}, \mathrm{~T}$ & $\mathrm{~S}, \mathrm{~A}, \mathrm{~T}, \mathrm{~b}$ & $\mathrm{~S}, \mathrm{~A}, \mathrm{~T}, \mathrm{~b}$ \\
\hline 00133 & M1470/7: & vl 1,2 & $\mathrm{~S} 1,2, \mathrm{~T}$ & $\mathrm{~S}, \mathrm{~A}, \mathrm{~T}, \mathrm{~b}$ & \\
\hline 00123 & M1470/8: & & $\mathrm{S} 1,2, \mathrm{~A}, \mathrm{~T}$ & $\mathrm{~S}, \mathrm{~A}, \mathrm{~T}, \mathrm{~b}$ & $\mathrm{~S}, \mathrm{~A}, \mathrm{~T}, \mathrm{~b} ; \mathrm{S} 1,2^{48}$ \\
\hline 00282 & M1470/9: & vl 1,2 & $\mathrm{~S} 1,2, \mathrm{~A}, \mathrm{~T}$ & & \\
\hline 00011 & M1470/12: & vl 1,2 & $\mathrm{~S} 1,2, \mathrm{~A}, \mathrm{~T}$ & & \\
\hline 00047 & M1471/1: & vl 1,2 & $\mathrm{~S} 1,2, \mathrm{~A}, \mathrm{~T}$ & $\mathrm{~S}, \mathrm{~A}, \mathrm{~T}, \mathrm{~b}$ & $\mathrm{~S}, \mathrm{~A}, \mathrm{~T}, \mathrm{~b}$ \\
\hline 00075 & M1471/2: & & $\mathrm{S}, \mathrm{A}, \mathrm{T}, \mathrm{b}$ & $\mathrm{S}, \mathrm{A}, \mathrm{T}, \mathrm{b}$ & \\
\hline 00232 & M1471/3: & & S $1,2, A, T$ & $\mathrm{~S}, \mathrm{~A}, \mathrm{~T}, \mathrm{~B} ;{ }^{49}$ & \\
\hline 00045 & M1471/5: & vl 1,2 & $\mathrm{~S} 1,2, \mathrm{~A}, \mathrm{~T} 1,2$ & $\mathrm{~S}, \mathrm{~A}, \mathrm{~T}, \mathrm{~b}$ & \\
\hline 00209 & M1471/8: & & $\mathrm{S} 1,2, \mathrm{~A}, \mathrm{~T}$ & $\mathrm{~S}, \mathrm{~A}, \mathrm{~T}, \mathrm{~b}$ & $\mathrm{~S}, \mathrm{~A}, \mathrm{~T}, \mathrm{~b}$ \\
\hline 00142 & M1471/11: & vl 1,2 & S $1,2, \mathrm{~A}, \mathrm{~T}$ & $\mathrm{~S}, \mathrm{~A}, \mathrm{~T}, \mathrm{~b}$ & $\mathrm{~S}, \mathrm{~A}, \mathrm{~T}, \mathrm{~b}$ \\
\hline - & M1471/13: & vl $1,2^{50}$ & S $1,2, \mathrm{~A}, \mathrm{~T} ; 1,2$ & $\mathrm{~S}, \mathrm{~A}, \mathrm{~T}, \mathrm{~b}$ & \\
\hline
\end{tabular}

32. Cobles $1 .^{\mathrm{a}}$ i $3 .^{\mathrm{a}}=\mathrm{T}$; C $2 .^{\mathrm{a}}=\mathrm{S} 1$; altres $3 \mathrm{i}$ "recit" ${ }^{\circ}$ " A.

33. Estribillo amb "solos" per les diferents veus.

34. El Recitatiu el fa el S 1.

35. Solo de $\mathrm{S} 1+$ totes les veus.

36. Cobles $1 .^{\mathrm{a}}$ i $3 .^{\mathrm{a}}: \mathrm{S} 1+4$ veus; $2 .^{\mathrm{a}}$ i $4 .^{\mathrm{a}}: \mathrm{T}+4$ veus.

37. Recitatiu i Âria $=$ A.

38. Recitatiu $=\mathrm{S} 1$.

39. Recitatiu $=\mathrm{A}$, acompanyament.

40. Cobles (o: "desp..$^{\mathrm{s}}$ del Recita $\left.{ }^{\text {do }}\right)=$ A, violí 1, 2, acompanyament als violins, acompanyament.

41. Recitatiu $=\mathrm{S} 2$, acompanyament.

42. Ària $=S 2$, violí 1,2 , acompanyament als violins, acompanyament.

43. Cobles $1 .^{\mathrm{a}}$ i 4. ${ }^{\mathrm{a}} \mathrm{S} \mathrm{S} 1 ; 2 .^{\mathrm{a}}: \mathrm{T} ; 3 .^{\mathrm{a}}: \mathrm{A}+$ violí 1,2 , acompanyament.

44. Recitatiu $=\mathrm{T}$, acompanyament.

45. Ària $=\mathrm{T}$, violí 1,2 , acompanyament.

46. Estructura més aviat semblant a la Cantata.

47. Els "Bajos" són instruments, mentre no es digui el contrari. Així, doncs, l'escriuré en minúscula. a 15 ".

48. Al manuscrit diu: "Tiple $1 .^{\circ} 4 .^{\circ}$ Coro", "A 15 Chirimia", "Tiple $2 .^{\circ} 4 .^{\circ}$ Coro", "A 15 Chirimia" i "Sacapuche $4 .^{\circ}$ Coro

49. Aquest "Bajo" dúu el text escrit, per tant, és veu humana i, en conseqüència, li correspon inicial majúscula. 50. "3. Coro". 


\begin{tabular}{|c|c|c|c|c|c|}
\hline - & M1471/14: & vl $1,2^{51}$ & $\mathrm{~S}^{52} 1,2, \mathrm{~A}, \mathrm{~T}$ & $\mathrm{~S}, \mathrm{~A}, \mathrm{~T}, \mathrm{~b}$ & $\mathrm{~S}, \mathrm{~A}, \mathrm{~T}, \mathrm{~b}$ \\
\hline - & M1471/15: & & $\mathrm{S} 1, \mathrm{~A}, \mathrm{~T}$ & $\mathrm{~S}, \mathrm{~A}, \mathrm{~T}, \mathrm{~b}$ & $\mathrm{~S}, \mathrm{~A}, \mathrm{~T}, \mathrm{~b}$ \\
\hline- & M1471/16: & & $\mathrm{S} 1,2, \mathrm{~A}, \mathrm{~T}$ & $\mathrm{~S}, \mathrm{~A}, \mathrm{~T}, \mathrm{~b}$ & $\mathrm{~S}, \mathrm{~A}, \mathrm{~T}, \mathrm{~b} ; \mathrm{s} 1,2, \mathrm{~b}^{53}$ \\
\hline 00037 & M1471/17: & vl 1,2 xeremia $1^{54}, 2$ & S 1,2 & $\mathrm{~S}, \mathrm{~A}, \mathrm{~T}, \mathrm{~b}$ & $\mathrm{~S}, \mathrm{~A}, \mathrm{~T}, \mathrm{~b} ; \mathrm{b} 1^{55}, 2$ \\
\hline - & M1471/18: & vl $1^{56}, 2$ xeremia 1,2 & $\mathrm{~S} 1,2, \mathrm{~A}, \mathrm{~T}$ & $\mathrm{~S}, \mathrm{~A}, \mathrm{~T}, \mathrm{~b}$ & $\mathrm{~S}, \mathrm{~A}, \mathrm{~T}, \mathrm{~b} ; \mathrm{b} 1,2$ \\
\hline - & M1471/19: & & $\mathrm{S} 1,2, \mathrm{~A}, \mathrm{~T}$ & $\mathrm{~S}, \mathrm{~A}, \mathrm{~T}, \mathrm{~b}$ & $\mathrm{~S}, \mathrm{~A}, \mathrm{~T}, \mathrm{~b}$ \\
\hline 00256 & M1471/20: & vl 1,2 xeremia $1^{57}$ & S $1,2, A, T$ & $\mathrm{~S}, \mathrm{~A}, \mathrm{~T}, \mathrm{~b}$ & $\mathrm{~S}, \mathrm{~A}, \mathrm{~T}, \mathrm{~b}$ \\
\hline - & M1471/21: & & $\mathrm{S} 1,2 \mathrm{~T} 1,2$ & $\mathrm{~S}, \mathrm{~A}, \mathrm{~T}, \mathrm{~b}$ & $\mathrm{~S}, \mathrm{~A}, \mathrm{~b}^{58}$ \\
\hline 00019 & M1472 /7: & & $\mathrm{S} 1,2, \mathrm{~A}, \mathrm{~T} 1,2$ & $\mathrm{~S}, \mathrm{~A}, \mathrm{~T}$ & $\mathrm{~S}, \mathrm{~A}, \mathrm{~T}, \mathrm{~b}$ \\
\hline 00231 & M1472 /8: & & $\mathrm{S} 1,2, \mathrm{~A}, \mathrm{~T}$ & $\mathrm{~S}, \mathrm{~A}, \mathrm{~T}, \mathrm{~b}$ & $\mathrm{~S}, \mathrm{~A}, \mathrm{~T}, \mathrm{~b} ; \mathrm{s} 1,2, \mathrm{~b}^{59}$ \\
\hline 00254 & M1472 /9: & vl 1,2 xeremia 1,2 & $\mathrm{~S} 1,2, \mathrm{~A}, \mathrm{~T} 1,2$ & $\mathrm{~S}, \mathrm{~A}, \mathrm{~T}, \mathrm{~b}$ & $\mathrm{~S}, \mathrm{~A}, \mathrm{~T}, \mathrm{~b}$ \\
\hline 00040 & M1472 /10: & vl $1,2^{60}$ xeremia & $\mathrm{S} 1,2, \mathrm{~A}, \mathrm{~T}$ & $\mathrm{~S}, \mathrm{~A}, \mathrm{~T}, \mathrm{~b}$ & $\mathrm{~S}, \mathrm{~A}, \mathrm{~T}, \mathrm{~b}$ \\
\hline 00260 & M1472 /11: & vl $1,2^{61}$ xeremia & $\mathrm{S}, \mathrm{A}, \mathrm{T}, \mathrm{b}$ & $\mathrm{S}, \mathrm{A}, \mathrm{T}, \mathrm{b}$ & \\
\hline - & M1472 /12: & vl 1,2 & $\mathrm{~S} 1,2, \mathrm{~A}, \mathrm{~T}$ & & \\
\hline 00036 & M1472 /13: & vl 1,2 & $\mathrm{~S} 1,2, \mathrm{~A}, \mathrm{~T}$ & & \\
\hline 00246 & M1472 /14: & vl 1,2 & $\mathrm{~S} 1,2, \mathrm{~A}, \mathrm{~T}$ & & \\
\hline 00281 & M1472 /15: & vl 1,2 & $\mathrm{~S} 1,2, \mathrm{~A}, \mathrm{~T}$ & & \\
\hline - & M1472 /16: & vl 1,2 & $\mathrm{~S} 1,2, \mathrm{~A}, \mathrm{~T}$ & & \\
\hline 00115 & M1472 /17: & vl 1,2 & $\mathrm{~S} 1,2, \mathrm{~A}, \mathrm{~T}$ & & \\
\hline 00317 & M1472 /18: & vl 1,2 & $\mathrm{~S} 1,2, \mathrm{~A}, \mathrm{~T}$ & & \\
\hline - & M1472 /19: & vl 1,2 & S $1,2, A, T$ & & \\
\hline 00303 & M1472 /20: & vl 1,2 & $\mathrm{~S} 1,2, \mathrm{~A}, \mathrm{~T}$ & & \\
\hline - & M1473 /9: & vl 1,2 & $\mathrm{~S} 1,2, \mathrm{~A}, \mathrm{~T}$ & & \\
\hline 00067 & M1473 /10: & vl 1,2 & $\mathrm{~S} 1,2, \mathrm{~A}, \mathrm{~T}$ & & \\
\hline 00034 & M1474 /18: & vl 1,2 & $\mathrm{~S} 1,2, \mathrm{~A}, \mathrm{~T}$ & & \\
\hline
\end{tabular}

\begin{tabular}{|c|c|c|c|c|c|c|}
\hline N. ${ }^{\circ}$ & Sign. & acompanyan & & & & \\
\hline 00072 & M1470/2: & als violins & a les xeremies 1 & & orgue, continu & \\
\hline 00073 & M1470/5: & & & & orgue, continu & \\
\hline 00313 & M1470/6: & & & sacabuche & orgue, continu & \\
\hline 00133 & M1470/7: & violó & & & & acompanyament \\
\hline 00123 & M1470/8: & & & sacapuche & orgue, continu & \\
\hline 00282 & M1470/9: & als violins & & & & acompanyament \\
\hline 00011 & M1470/12: & violó & & & & acompanyament \\
\hline 00047 & M1471/1: & & & & orgue, continu & \\
\hline
\end{tabular}

51. El grup de violins i violó, el defineix com " $3 .^{\circ}$ Coro $^{\mathrm{a}}$. (Altres vegades també).

52. "1. "Coro".

53. És un quart Coro d'instruments, "termino de ministriles".

54. "Tiple 1. ${ }^{\circ}$ de Chirimias".

55. "Tiple 1. ' de Sacapuche".

56. "Ayroso". A les xeremies i sacapuches: "Tiple 1. de Chirimias". "Tiple 1. de Sacapuche".

57. "Tiple de Chirimias".

58. És un baix instrumental.

59. Coro de violins i violó, o acompanyament als violins.

60. "4. " Coro".

61. Anomena "2. ${ }^{\circ}$ Coro" al grup de "chirimia", "violines [1, 2]", "Violon" i el "Bajo". Diu que el "Villancico" és de "5. tono", encara que acaba amb la nota carácterística del to 11t o Joni, això és, el Do. Hi ha, d'extraordinari, un "acomp. to al arpa al Coro de los violines" i un altre "Al Arpa. A 16", amb el contingut musical diferent, el qual detall fa pensar, realment, en dues arpes. 


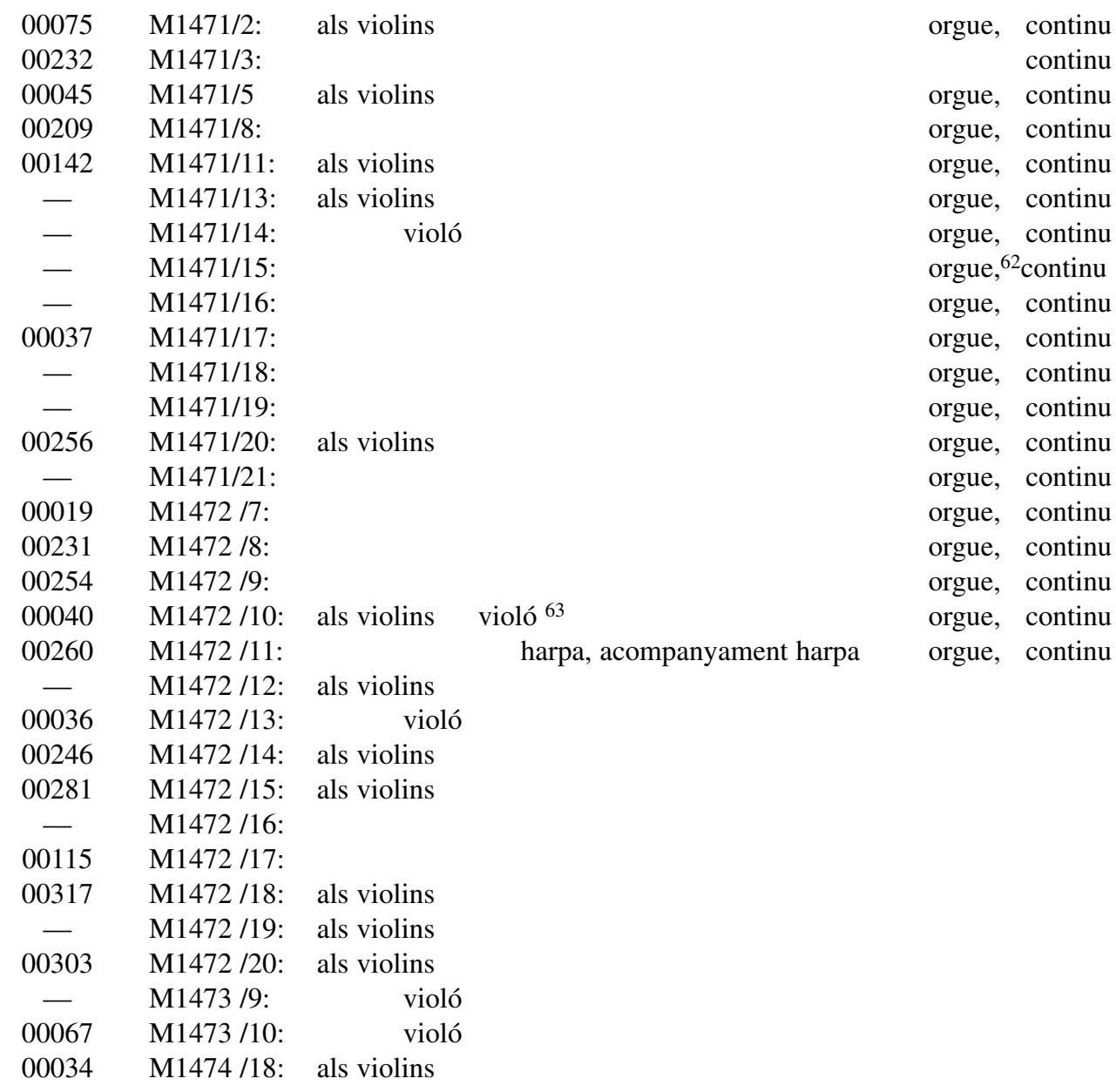

acompanyament acompanyament acompanyament acompanyament acompanyament acompanyament acompanyament acompanyament acompanyament acompanyament acompanyament acompanyament

\section{II) Tonos}

\begin{tabular}{|c|c|c|c|c|c|}
\hline N. ${ }^{\circ}$ RISM & Sign. $E$ : $B b c$ & Instrum. $^{\mathrm{s}}$ & Veus & & acompanyaments \\
\hline 00160 & M1470/13 & vl 1,2 & $\mathrm{~S} 1,2, \mathrm{~A}, \mathrm{~T}$ & & acompanyament als violins, \\
\hline 00128 & M1611/26 & vl 1,2 & S 1,2 & violó, & acompanyament \\
\hline 00093 & M1611/27 & $\mathrm{vl}$ & A & & bc.fig \\
\hline 00158 & M1611/28 & & $\mathrm{S} 1,2, \mathrm{~A}, \mathrm{~T}$ & & bc.fig \\
\hline 00057 & M1611/29 & vl 1,2 & $\mathrm{~S}, \mathrm{~A}$ & & bc.fig \\
\hline 00001 & M1611/30 & vl 1,2 & $\mathrm{~S}$ & violó, & acompanyament \\
\hline 00035 & M1611/31 & vl 1,2 & $\mathrm{~S} 1,2,3, \mathrm{~T}$ & violó, & acompanyament \\
\hline 00050 & M1611/33 & & $\mathrm{S}$ & & acompanyament \\
\hline
\end{tabular}

62. "Bolado", "espacio".

63. En aquesta ocasió diu: "violon" i també "acompañamiento a los Violines". 


\begin{tabular}{|c|c|c|c|c|c|c|}
\hline 00032 & M1611/34 & vl 1,2 & $\mathrm{~S}, \mathrm{~T}$ & & acompanyament als violins, & bc.fig \\
\hline 00151 & M1611/36 & & S & violó & acompanyament & \\
\hline 00213 & M1611/37 & vl 1,2 & $\mathrm{~S} 1,2, \mathrm{~A}, \mathrm{~T}$ & & acompanyament als violins, & acompanyament \\
\hline 00079 & M1611/38 & vl 1,2 & $\mathrm{~S} 1,2, \mathrm{~A}, \mathrm{~T}$ & & bc.fig & \\
\hline 00046 & M1611/39 & & $\mathrm{S} 1,2, \mathrm{~A}, \mathrm{~T}$ & & bc.fig & \\
\hline 00299 & M1611/40 & vl 1,2 & $\mathrm{~A} 1,2$ & & bc.fig & \\
\hline 00009 & M1611/41 & vl 1,2 & $\mathrm{~S} 1,2, \mathrm{~A}, \mathrm{~T}$ & & acompanyament als violins, & acompanyament \\
\hline 00124 & M1611/42 & vl 1 & $S$ & violó & acompanyament & \\
\hline 00283 & M1611/4364 & vl 1,2 & $\mathrm{~T}$ & & acompanyament als violins, & acompanyament \\
\hline 00255 & M1637/VIII/8 & $\mathrm{vl}$ & A & & bc.fig & \\
\hline 00253 & M1637/VIII/9 & vl 1,2 & S ("a solo y a 3" & & acompanyament & \\
\hline 00092 & M1686/26 & & $\mathrm{S}$ & & acompanyament & \\
\hline 00300 & M1686/27 & & $\mathrm{S}$ & & acompanyament & \\
\hline 00304 & M1686/28 & vl 1,2 & $\mathrm{~S}, \mathrm{~T}$ & violó & & \\
\hline 00239 & M1686/29 & vl 1,2 & $\mathrm{~S} 1,2, \mathrm{~A}, \mathrm{~T}$ & & acompanyament als violins, & acompanyament \\
\hline 00060 & M1686/30 & & $\mathrm{S} 1,2, \mathrm{~A}, \mathrm{~T}$ & & bc.fig & \\
\hline 00120 & M1686/31 & vl 1,2 & $\mathrm{~S}$ & & acompanyament als violins, & acompanyament \\
\hline 00095 & M1686/32 & & $\mathrm{S} 1,2, \mathrm{~A}, \mathrm{~T}$ & & bc.fig & \\
\hline 00270 & M1686/33 & & $\mathrm{S} 1,2, \mathrm{~A}, \mathrm{~T}$ & & bc.fig & \\
\hline 00159 & M1686/34 & vl 1,2 & $\mathrm{~S} 1,2, \mathrm{~A}, \mathrm{~T}$ & & acompanyament & \\
\hline 00271 & M1686/35 & vl 1,2 & $\mathrm{~S}, \mathrm{~T}$ & & acompanyament & \\
\hline 00110 & M1686/36 & vl 1,2 & $\mathrm{~S} 1,2, \mathrm{~A}, \mathrm{~T}$ & & acompanyament & \\
\hline 00105 & M1686/37 & & S1, 2, 3; Cupido & & $\mathrm{x}$ & \\
\hline 00114 & M1686/38 & vl 1,2 & $\mathrm{~S}$ & & acompanyament. & \\
\hline 00259 & M1686/39 & vl 1,2 & $\mathrm{~S} 1,2, \mathrm{~A}, \mathrm{~T}$ & & acompanyament als violins, & acompanyament \\
\hline 00113 & M1686/40 & vl 1,2 & $\mathrm{~S} 1,2, \mathrm{~A}, \mathrm{~T}$ & & acompanyament als violins, & acompanyament \\
\hline 00314 & M1686/41 & vl 1,2 & $\mathrm{~S} 1,2, \mathrm{~A}, \mathrm{~T}$ & & acompanyament als violins, & acompanyament \\
\hline 00112 & M1686/43 & vl 1,2 & $\mathrm{~S} 1,2, \mathrm{~A}, \mathrm{~T}$ & violó, & acompanyament & \\
\hline 00240 & M1686/44 & vl 1,2 & $\mathrm{~S} 1,2, \mathrm{~A}, \mathrm{~T}$ & violó, & acompanyament & \\
\hline - & M1690/2 & vl 1,2 & {$[\mathrm{~S}], \mathrm{A}, \mathrm{T}$} & & acompanyament als violins, & acompanyament \\
\hline- & PL: $K j$, f. 7 & & $\mathrm{~S} 1,2, \mathrm{~A}, \mathrm{~T}$ & & acompanyament & \\
\hline
\end{tabular}

\section{Esquema F) Tonalitat $^{65}$}

\section{I) Villancets}

\begin{tabular}{cllll} 
N. $^{\mathbf{0}}$ RISM & Sign. $\boldsymbol{E}: \boldsymbol{B} \boldsymbol{b} \boldsymbol{c}$ & Tonalitat & & \\
\hline 00072 & M1470/2: & acaba en Si+b & $5 \mathrm{tt}$ & Lidi $^{67}$ \\
00073 & M1470/5: & acaba en Do & $11 \mathrm{t}$ & Joni
\end{tabular}

64. Estructura més aviat semblant a la Cantata.

65. Tant en els Villancicos, com en els Tonos, Valls empra majorment les modalitats tradicionals eclesiàstiques, algunes transportades, com si hi jogués i les tonalitats modernes. En referir-me a aquests Modes, ho faig de manera conjunta. Enteneu, doncs, el To autèntic, però també el seu corresponent plagal. La correspondència de les tonalitats antigues-modernes està tractada molt clarament pel Dr. A. Ezquerro, a la seva tesi doctoral, La música vocal en Aragón en el segundo tercio del siglo XVII, vol. II, pp. 313-314.

66. S'entén a la clau. 


\begin{tabular}{|c|c|c|c|c|}
\hline 00313 & M1470/6: & acaba en $\mathrm{Fa}$ & $5 \mathrm{t}$ & Lidi \\
\hline 00133 & M1470/7: & acaba en LaM (3\#) & A & \\
\hline 00123 & M1470/8: & acaba en $\mathrm{Fa}+\mathrm{b}^{68}$ & $11 \mathrm{tt}$ & Joni \\
\hline 00282 & M1470/9: & acaba en $\mathrm{Re}$ & $1 \mathrm{t}$ & Dori \\
\hline 00011 & M1470/12: & acaba en LaM (3\#) & A & \\
\hline 00047 & M1471/1: & acaba en Sol & $7 \mathrm{t}$ & Mixolidi \\
\hline 00075 & M1471/2: & acaba en SibM (2b) & B & \\
\hline 00232 & M1471/3: & acaba en Sol+b & $1 \mathrm{tt}$ & Dori \\
\hline 00045 & M1471/5: & acaba en Fa+b & $11 \mathrm{tt}$ & Joni \\
\hline 00209 & M1471/8: & acaba en $\mathrm{Fa}+\mathrm{b}$ & $11 \mathrm{tt}$ & Joni \\
\hline 00142 & M1471/11: & acaba en Mi & $3 t$ & Frigi ${ }^{69}$ \\
\hline - & M1471/13: & acaba en $\mathrm{Si}+\mathrm{b}$ & $5 \mathrm{tt}$ & Lidi \\
\hline - & M1471/14: & acaba en $\mathrm{Fa}$ & $5 \mathrm{t}$ & Lidi \\
\hline - & M1471/15: & acaba en $\mathrm{Fa}+\mathrm{b}$ & $11 \mathrm{tt}$ & Joni \\
\hline - & M1471/16: & acaba en $\mathrm{Fa}$ & $5 \mathrm{t}$ & Lidi \\
\hline 00037 & M1471/17: & acaba en $\mathrm{Fa}+\mathrm{b}$ & $11 \mathrm{tt}$ & Joni \\
\hline - & M1471/18: & acaba en MibM (3b) & E & \\
\hline- & M1471/19: & acaba en $\mathrm{Fa}+\mathrm{b}$ & $11 \mathrm{tt}$ & Joni \\
\hline 00256 & M1471/20: & acaba en $\mathrm{Si}+\mathrm{b}$ & $5 \mathrm{tt}$ & Lidi \\
\hline - & M1471/21: & acaba en $\mathrm{Fa}+\mathrm{b}$ & $11 \mathrm{tt}$ & Joni \\
\hline 00019 & M1472 /7: & acaba en $\mathrm{Si}+\mathrm{b}$ & $5 \mathrm{tt}$ & Lidi \\
\hline 00231 & M1472 /8: & acaba en Sol & $7 \mathrm{t}$ & Mixolidi \\
\hline 00254 & M1472 /9: & acaba en Do & $11 \mathrm{t}$ & Joni \\
\hline 00040 & M1472 /10: & acaba en $\mathrm{Si}+\mathrm{b}$ & $5 \mathrm{tt}$ & Lidi \\
\hline 00260 & M1472 /11: & acaba en Do & $5 \mathrm{t}$ & Lidi $^{70}$ \\
\hline - & M1472 /12: & acaba en ReM (2\#) & $\mathrm{D}$ & \\
\hline 00036 & M1472 /13: & acaba en Do & $11 \mathrm{t}$ & Joni \\
\hline 00246 & M1472 /14: & acaba en ReM (2\#) & D & \\
\hline 00281 & M1472 /15: & acaba en Do & $11 \mathrm{t}$ & Joni \\
\hline - & M1472 /16: & acaba en Mim (1\#) & E & \\
\hline 00115 & M1472 /17: & acaba en Do & $11 \mathrm{t}$ & Joni \\
\hline 00317 & M1472 /18: & acaba en $\mathrm{Si}+\mathrm{b}$ & $5 \mathrm{tt}$ & Lidi \\
\hline- & M1472 /19: & acaba en $\mathrm{Si}+\mathrm{b}$ & $5 \mathrm{tt}$ & Lidi \\
\hline 00303 & M1472 /20: & acaba en Sol+b & $1 \mathrm{tt}$ & Dori \\
\hline- & M1473 /9: & acaba en SolM (1\#) & G & \\
\hline 00067 & M1473 /10: & acaba en ReM (2\#) & $\mathrm{D}$ & \\
\hline 00034 & M1474 /18: & acaba en $\mathrm{Si}+\mathrm{b}$ & $5 \mathrm{tt}$ & Lidi $^{71}$ \\
\hline
\end{tabular}

67. En referir-me a aquests Modes, ho faig de manera conjunta. Enteneu, doncs, el To autèntic, però també el seu corresponent plagal.

68. acompanyament continu: clau de Do en quarta línia.

69. Cadència: La-Mi.

70. Valls diu: "5. ${ }^{\circ}$ tono".

71. Porta la data de 1702, com la Missa "Scala Aretina". 


\section{II) Tonos}

\begin{tabular}{|c|c|c|c|c|}
\hline N. ${ }^{\circ}$ RISM & Sign. $E: B b c$ & Tonalitat & & \\
\hline 00128 & M1611/26 & acaba en $\mathrm{Si}+\mathrm{b}$ & $5 \mathrm{tt}$ & Lidi \\
\hline 00093 & M1611/27 & acaba en Mi (4\#) & $11 \mathrm{t}$ & Joni $^{72}$ \\
\hline 00158 & M1611/28 & acaba en $\mathrm{Si}+\mathrm{b}$ & $5 \mathrm{tt}$ & Lidi \\
\hline 00057 & M1611/29 & acaba en Mi (3b) & $7 t$ & Mixolidi ${ }^{73}$ \\
\hline 00001 & M1611/30 & acaba en Do & $11 \mathrm{t}$ & Joni \\
\hline 00035 & M1611/31 & acaba en Do (1b) & $7 \mathrm{tt}$ & Mixolidi \\
\hline 00050 & M1611/33 & acaba en La & $9 t$ & Eoli \\
\hline 00032 & M1611/34 & acaba en $\mathrm{Si}+\mathrm{b}$ & $5 \mathrm{tt}$ & Lidi \\
\hline 00151 & M1611/36 & acaba en $\mathrm{Fa}+\mathrm{b}$ & $11 \mathrm{tt}$ & Joni \\
\hline 00213 & M1611/37 & $\mathrm{acaba}$ en $\mathrm{Fa}+\mathrm{b}$ & $11 \mathrm{tt}$ & Joni \\
\hline 00079 & M1611/38 & $\mathrm{acaba}$ en $\mathrm{Fa}+\mathrm{b}$ & $11 \mathrm{tt}$ & Joni \\
\hline 00046 & M1611/39 & acaba en Sol+b & $1 \mathrm{tt}$ & Dori \\
\hline 00299 & M1611/40 & acaba en Do & $1 \mathrm{t}$ & Dori $^{74}$ \\
\hline 00009 & M1611/41 & acaba en $\mathrm{Mi}$ & $9 \mathrm{t}$ & Eoli $^{75}$ \\
\hline 00124 & M1611/42 & acaba en $\mathrm{Si}$ & $1 \mathrm{t}$ & Dori $^{76}$ \\
\hline 00283 & M1611/43 & acaba en Sol & $1 \mathrm{t}$ & Dori $^{77}$ \\
\hline 00255 & M1637/VIII/8 & acaba en $\mathrm{Fa}+\mathrm{b}$ & $11 \mathrm{tt}$ & Joni \\
\hline 00253 & M1637/VIII/9 & acaba en La+b & $3 \mathrm{tt}$ & Frigi \\
\hline 00092 & M1686/26 & acaba en $\mathrm{Fa}+\mathrm{b}$ & $11 \mathrm{tt}$ & Joni \\
\hline 00300 & M1686/27 & acaba en Sol & $7 t$ & Mixolidi \\
\hline 00304 & M1686/28 & acaba en La (3\#) & $11 \mathrm{tt}$ & Joni ${ }^{78}$ \\
\hline 00239 & M1686/29 & acaba en $\mathrm{Fa}+\mathrm{b}$ & $11 \mathrm{tt}$ & Joni \\
\hline 00060 & M1686/30 & acaba en $\mathrm{Si}+\mathrm{b}$ & $5 \mathrm{tt}$ & Lidi \\
\hline 00120 & M1686/31 & acaba en $\operatorname{Mi}(3 b)$ & $11 \mathrm{tt}$ & Joni $^{79}$ \\
\hline 00095 & M1686/32 & acaba en $\mathrm{Si}+\mathrm{b}$ & $5 \mathrm{tt}$ & Lidi \\
\hline 00270 & M1686/33 & acaba en $\mathrm{Re}$ & $1 \mathrm{t}$ & Dori \\
\hline 00159 & M1686/34 & acaba en $\mathrm{Re}$ & $1 \mathrm{t}$ & Dori \\
\hline 00271 & M1686/35 & acaba en $\operatorname{Mi}(3 b)$ & $11 \mathrm{tt}$ & Joni ${ }^{80}$ \\
\hline 00110 & M1686/36 & $\mathrm{acaba}$ en $\mathrm{Fa}+\mathrm{b}$ & $11 \mathrm{tt}$ & Joni \\
\hline 00105 & M1686/37 & acaba en Sol+b & $1 \mathrm{tt}$ & Dori \\
\hline 00114 & M1686/38 & acaba en Sol(1\#) & G & \\
\hline 00259 & M1686/39 & acaba en $\mathrm{Si}+\mathrm{b}$ & $5 \mathrm{tt}$ & Lidi \\
\hline 00113 & M1686/40 & acaba en $\mathrm{Re}$ & $1 \mathrm{t}$ & Dori \\
\hline 00314 & M1686/41 & acaba en $\operatorname{Re}$ & $1 \mathrm{t}$ & Dori \\
\hline 00112 & M1686/43 & acaba en $\mathrm{Mi}(4 \#)$ & $\mathrm{E}$ & \\
\hline 00240 & M1686/44 & acaba en $\operatorname{Re}$ & $1 \mathrm{t}$ & Dori \\
\hline
\end{tabular}

72. Transportat $3 .^{\mathrm{a}}$ major alta.

73. Transportat $3 .^{a}$ menor alta.

74. Transportat un to baix.

75. Transportat una quarta baixa.

76. Transportat una tercera menor baixa

77. Transportat una quarta alta.

78. Transportat una tercera menor baixa.

79. Transportat una tercera menor alta.

80. Transportat una tercera menor alta. 


$\begin{array}{lllll}00160 & \text { M1470/13 } & \text { acaba en } \operatorname{Re}(2 \#) & \text { D } & \\ - & \text { M1690/2 } & \text { acaba en Fa+b } & 11 \text { tt } & \text { Joni } \\ - & \text { PL: } K j, \text { f. } 7 & \text { acaba en } S i+b & 5 t t & \text { Lidi }\end{array}$

\section{Esquema G) Sinopsi de l'}

\begin{tabular}{|c|c|c|}
\hline Esquema formal & Villancets & Tonos \\
\hline$?-\mathrm{C}$ & & 2 \\
\hline $\mathrm{E}-\mathrm{C}$ & 22 & 26 \\
\hline $\mathrm{E}-\mathrm{A}-\mathrm{C}$ & & 1 \\
\hline$E-C-R$ & & 2 \\
\hline $\mathrm{E}-\mathrm{R}-\mathrm{C}$ & & 1 \\
\hline $\mathrm{E}-\mathrm{C}-2 .^{\circ} \mathrm{E}$ & & 1 \\
\hline I - E - C & 7 & \\
\hline $\mathrm{I}-\mathrm{R}-\mathrm{T}$ & 1 & \\
\hline$A-R-C-T$ & 1 & \\
\hline$E-R-A-C$ & 2 & 1 \\
\hline$E-R-A-T$ & 1 & \\
\hline I - E - C - R & 1 & \\
\hline I - E - R - A & 1 & \\
\hline$E-C-T-R-A$ & & 1 \\
\hline$E-R-A-R-A$ & 1 & \\
\hline$E-R-A-R-T$ & & 1 \\
\hline$E-R-C-R-A$ & & 1 \\
\hline $\mathrm{I}-\mathrm{R}-\mathrm{A}-\mathrm{R}-\mathrm{A}$ & & 1 \\
\hline I - E - R - A - R - A & 1 & \\
\hline $\mathrm{E}-\mathrm{R}$ - post $\mathrm{R}-\mathrm{A}-\mathrm{R}$ - post $\mathrm{R}-\mathrm{A}$ & & 1 \\
\hline $\mathrm{E}-\mathrm{R}-\mathrm{A}-\mathrm{R}-\mathrm{A}-\mathrm{R}-\mathrm{A}-\mathrm{R}-\mathrm{A}$ & 1 & \\
\hline
\end{tabular}

81. Aquesta Signatura es diu: “M1690/2: Tonos" i conté diferents Tonos incomplets. Al Tono que m'ocupa, sembla que només li manca el Tiple. 


\section{Esquema H) Sinopsi de les:}

\begin{tabular}{lcr} 
Veus & Villancets & Tonos \\
\hline 1 (solo) & & 11 \\
2 (duo) & & 3 \\
3 & & 2 \\
4 & & 17 \\
5 & 3 & 2 \\
6 & 1 & \\
7 & 3 & \\
8 & 2 & \\
9 & 3 & \\
10 & 3 & \\
11 & 12 & \\
12 & 2 & \\
13 & 2 & \\
14 & 3 & \\
15 & 4 & \\
16 & 1 & Total: 39
\end{tabular}

\section{Esquema I) Sinopsi dels:}

\begin{tabular}{lcc} 
Coros $^{82}$ & Villancets & Tonos \\
\hline Solista & & 11 \\
1 Coro & 14 & 28 \\
2 Coros & 6 & \\
3 Coros & 14 & \\
4 Coros & 5 & Total: 39
\end{tabular}

82. Sovint computa els instruments com un Coro que pot ser el: "2.", "3. "”, "4." o " 5. .". 


\section{Esquema J) Sinopsi dels:}

\begin{tabular}{lrr} 
Instruments & Villancicos & Tonos \\
\hline un violí & 24 & 3 \\
dos violins & 5 & 24 \\
una xeremia & 5 & \\
dues xeremies & 1 & 12 \\
clarí & 15 & 8 \\
sense violins & 6 & 11 \\
violó & 12 & 1 \\
acompanyament als violins & 8 & \\
sacabutx & 1 & 38 \\
arpa & 30 & 20 \\
orgue & 37 & \\
acompanyament / acompanyament continu & 25 & \\
sense violó / acompanyament als violins & 12 & \\
sense instruments & &
\end{tabular}

\section{Esquema K) Sinopsi de les:}

\begin{tabular}{lcc} 
Dedicatòries & Villancets & Tonos \\
\hline Entrada de rey $^{83}$ & 1 & 6 \\
Immaculada $^{83}$ & 4 & 2 \\
Encarnación & 1 & \\
Para un velo & 1 & 11 \\
Sto. Tomas de Aquino & 25 & \\
Smo. Sto. & 25 & \\
A N. ${ }^{\text {a S. }}$ & 1 & 7 \\
SS. Reyes & 1 & 1 \\
Corpus "en dia de S. Juan" & 1 & 4 \\
A S. Rafael & 1 & 1 \\
A. Sta. Eulalia & 1 & 6 \\
A lo humano & & \\
Asunción & & 1 \\
San Joseph & & \\
Espíritu Santo & & \\
S. Antonio & & \\
S. Fco. Javier & &
\end{tabular}

83. Incloc els que diuen: "A la Pura Concepcion", "A la Concepcion" o altre semblant.

84. El M 1474/18 està dedicat, també, "A los SS. Reyes".

85. Es tracta dels Tonos: M 1611/36 i /37; M 1686/31, /33, /37, /38 i /41. 
Un element molt important del barroc, són els volums o contrastos. En aquest aspecte, Valls combina: Solos amb polifonia; sol encarregar una cobla a cada veu, excloent l'Alt o Contralt, quan hi ha d'altres veus; en un sol Tono, introdueix un personatge, això és: Cupido.

Esquema L) Sinopsi de les:

\begin{tabular}{lcc} 
Tonalitats & Villancets & Tonos \\
\hline Joni & 12 & 13 \\
Dori & 3 & 10 \\
Frigi & 1 & 1 \\
Lidi & 12 & 7 \\
Mixolidi & 2 & 3 \\
Eoli & 2 & 2 \\
A & 1 & \\
B & 3 & 1 \\
D & 2 & 1 \\
E & 1 & 1 \\
G & 1 & \\
& &
\end{tabular}

Total: 39

Total: 39 